\title{
Fuzzy Sliding Control with Non-linear Observer for Magnetic Levitation Systems
}

\author{
A. M. Benomair*, A. R. Firdaus, M. O. Tokhi \\ Department of Automatic Control and Systems Engineering \\ The University of Sheffield \\ Sheffield, UK \\ ambenomair1@sheffield.ac.uk
}

\begin{abstract}
Magnetic levitation (Maglev) systems make significant contribution to industrial applications due their reduced power consumption, increased power efficiency and reduced cost of maintenance. Common applications include Maglev power generation (e.g. wind turbine), Maglev trains and medical devices (e.g. magnetically suspended artificial heart pump). This paper proposes fuzzy sliding-mode controller 'FSMC' with a nonlinear observer been used to estimate the unmeasured states. Simulations are performed with nonlinear mathematical model of the Maglev system, and the results show that the proposed observer and control strategy perform well.
\end{abstract}

Index Terms-Magnetic levitation system; Nonlinear observer; Sliding-mode control; Fuzzy sliding-mode control;

\section{INTRODUCTION}

Magnetically levitated train constitutes one of the significant advance made due to Maglev technologies. The Japan's Maglev train has established world record train speeds of 581 $\mathrm{km} / \mathrm{h}$ in 2003 and $603 \mathrm{~km} / \mathrm{h}$ in 2014. Similarly, vertical axis wind turbine (VAWT) using magnetic bearing is able to start producing power with wind speeds as low as $1.5 \mathrm{~m} / \mathrm{s}$. Experimental results of Maglev (VAWT) in [1] show that system vibration can be reduced by $37.5 \%$, and power generating capability of the system is increased by $12 \%$ using Maglev concept. A combination of sliding mode control (SMC) with fuzzy logic is used in this work as, it is difficult to prove the stability of fuzzy logic controller (FSMC). Moreover, SMC is insensitive to external disturbance and to parameter variations, and able to decouple systems high-dimensional system into lower-dimensional sub-systems. This feature can help reduce the size of rule base of an FLC. Finally this combination can effectively alleviate the prevalent chattering problem of SMC [2].

Sliding-mode control is used in [3] to control the linearised magnetic ball levitation system over a fixed set of operating points using singular perturbation method. In this paper two kinds of traditional fuzzy sliding-mode control (FSMC) are first introduced, based on calculation of the control action using error / change of error $e / \dot{e}$ in the first instance and secondly the sliding function $S$. Then a novel fuzzy slidingmode control (NFSMC) is proposed in which the fuzzy natural control $u_{n f s}$ is calculated using sliding function with integration of an adjustable gain. The simulation results show the superior performance can be achieved to the NFSMC in comparison to the traditional FSMC.

A modified dynamic sliding-mode control has been reported in [4] to overcome the chattering problem of SMC, where the proposed controller is used to stabilize the dynamic of Maglev system in the new coordinates using feedback linearisation control. The results show that the modified dynamic SMC can provide smoother control action compared to classic dynamic SMC and up to $25 \%$ more robustness to parameter variations.

\section{NOnlinear Dynamic Model of MagleV System}

The Maglev system consider here serves to keep a small steel ball in stable levitation at some steady-state operating position. An electromagnet is used to produce forces to support the ball (see Fig. 1). The electromagnetic forces are related to the electrical current passing through the electromagnet coil;

$$
f_{\text {em }}=-\frac{1}{2} L_{0} x_{0}\left(\frac{i}{x}\right)^{2}
$$

where $f_{e m}$ is the electromagnetic forces, $L_{0}$ is the additional inductance, $x_{0}$ is an arbitrary of the object and $i$ is the coil current.

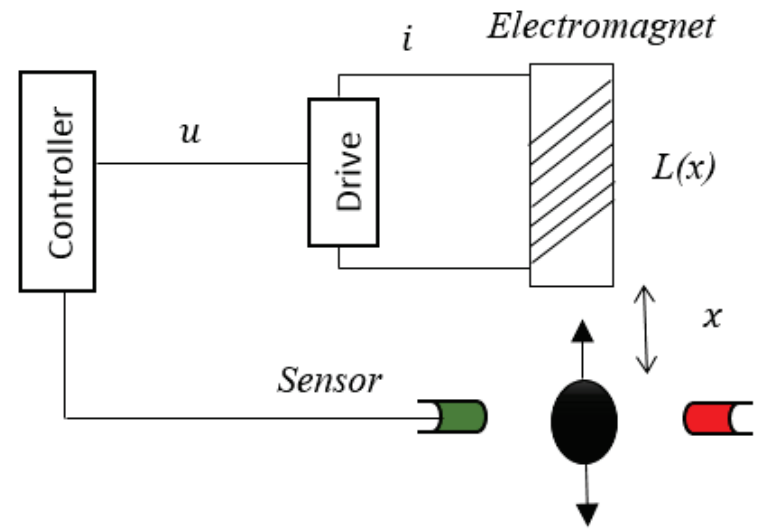

Fig. 1: Active magnetic levitation system (MLS) 
The object is suspended by balancing between the force of gravity and electromagnetic force. Applying Newtons 3rd law of motion, the dynamic form of the mechanical system can be written as

$$
\begin{aligned}
& f_{\text {net }}=f_{g}-f_{\text {em }} \\
& m \ddot{x}=m g+c\left(\frac{i}{x}\right)^{2}
\end{aligned}
$$

where, $f_{n e t}$ is the net force and $f_{g}$ is force due to gravity.

Define the states $x_{1}=x$ (position), $x_{2}=V$ (velocity), $x_{3}=i$ (current), the system input $u=v$ (applied voltage) and $c=\frac{1}{2} L_{0} x_{0}$. The nonlinear state space model of Maglev system can be expressed as

$$
\left[\begin{array}{c}
\dot{x}_{1} \\
\dot{x}_{2} \\
\dot{x}_{3}
\end{array}\right]=\left[\begin{array}{c}
x_{2} \\
g-\frac{c}{m}\left(\frac{x_{3}}{x_{1}}\right)^{2} \\
\frac{R}{L} x_{3}+\frac{2 c}{L}\left(\frac{x_{2} x_{3}}{x_{1}^{2}}\right)+\frac{1}{L} u
\end{array}\right]
$$

\section{SLIDING-MODE CONTROL}

The state variables for the magnetic ball levitation system are described as $\underline{x}=\left[\begin{array}{lll}x_{1} & x_{2} & x_{3}\end{array}\right]^{T}$, the position of the object, velocity and coil current. Define the vector of the desired values as $\underline{x}_{d}=\left[\begin{array}{lll}r_{1} & r_{2} & r_{3}\end{array}\right]^{T}$, where $r_{1}$ represents the desired position and $r_{2} \& r_{3}$ equal to zero. Then the vector of tracking error is defined as

$$
E=\left[\begin{array}{lll}
e & \dot{e} & \ddot{e}
\end{array}\right]=\left[\begin{array}{lll}
e_{1} & e_{2} & e_{3}
\end{array}\right]
$$

A sliding mode controller can be effectively applied to a nonlinear system in spite of parameter uncertainties and external disturbances. The dynamics of a nonlinear system can be described in the state space form as follows

$$
\dot{x}(t)=f(x ; t)+g(x ; t) u(t)
$$

For a control system, generally the sliding surface is selected as

$$
\sigma(x ; t)=S x(t)
$$

where $S$ is a matrix of positive constant elements with a dimension of $[m \times n]$. For a 3rd order system, the time varying surface $\sigma(t)$ can be defined as

$$
\sigma(t)=\left(\frac{d}{d t}+s\right)^{3-1} E
$$

where $s$ is strictly positive constant and indicates the slope of sliding surface. Thus from equation (7), the sliding surface can be written as

$$
\sigma(t)=\left[\begin{array}{lll}
s^{2} & 2 s & 1
\end{array}\right]\left[\begin{array}{l}
e_{1} \\
e_{2} \\
e_{3}
\end{array}\right]
$$

or it can be simplified as follows

$$
\sigma(t)=\left[\begin{array}{lll}
s_{1} & s_{2} & 1
\end{array}\right]\left[\begin{array}{l}
e_{1} \\
e_{2} \\
e_{3}
\end{array}\right]
$$

where $s_{2}=2 \sqrt{s_{1}}$

considering the dynamical system in equation (5), the developed control law is required to drive the system's trajectories towards the sliding surface in finite time $t \leq t_{r}$ and maintain motion on the surface " $\sigma=0$ " thereafter, in the presence of disturbance. A stability control law is designed using Lyapunov stability condition $\sigma^{T} \dot{\sigma}<0$. The general form of the control law for the sliding mode controller can be written as

$$
u=u_{e q}+u_{n}
$$

where $u_{e q}$ and $u_{n}$ are the equivalent control and natural control receptively. $u_{e q}$ can be interpreted as a continuous control law to maintain the dynamics of the system on both sides of the sliding surface " i.e $\dot{\sigma}=0$ " [5], while the natural control $u_{n}$ is discontinuous control law to be designed to account for nonzero uncertainties [6].

Substituting for $u(t)$ from equation (10) into equation (5), yields the closed-loop dynamics of the system as

$$
\dot{x}(t)=f(x ; t)+g(x)\left(u_{e q}+u_{n}\right)
$$

Since the necessary condition for the output trajectory to remain on the sliding surface $\sigma$ is that $\dot{\sigma}=0$ which expressed as $\dot{\sigma}=S \dot{x}(t)=0$. The equivalent control is augmented by auxiliary control effort termed as hitting the sliding surface and determined as

$$
u_{e q}=-(S \hat{g}(x))^{-1} S \hat{f}(x, t)
$$

The natural control is used to maintain the status trajectory on sliding surfaces using signum function which requires infinite switching on the part of actuator and the control signal, and this is expressed [7] as

$$
u_{n}=-K(S \hat{g}(x))^{-1} \operatorname{sign}(\sigma)
$$

\section{FuZZY SLIDING-Mode CONTROL}

The general structure of FSMC' is shown in Fig. 2.

The essential purpose of using this technique is to improve the switching function of natural control $\left(u_{n}\right)$ with fuzzy logic system. As the proposed method endeavours to eliminate the chattering phenomenon during sliding mode condition in the sliding surface.

The membership functions of fuzzy input $\left(U_{n}\right)$ and output $\left(F U_{n}\right)$ are created through the the values of sliding surface $(\sigma)$ as seen in Fig. 3, making use of fuzzy sets of seven 


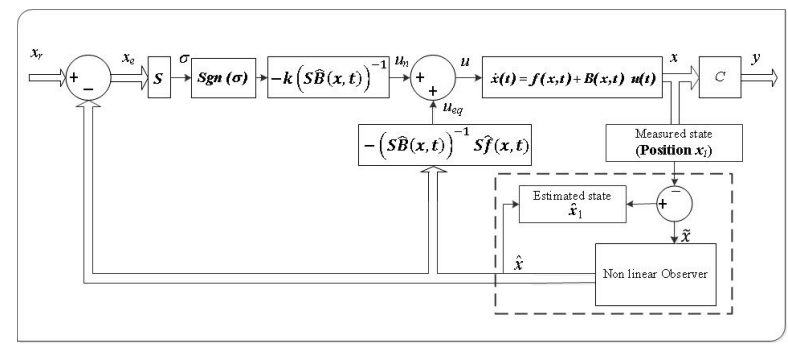

Fig. 2: Block diagram of Maglev system with SMC controller
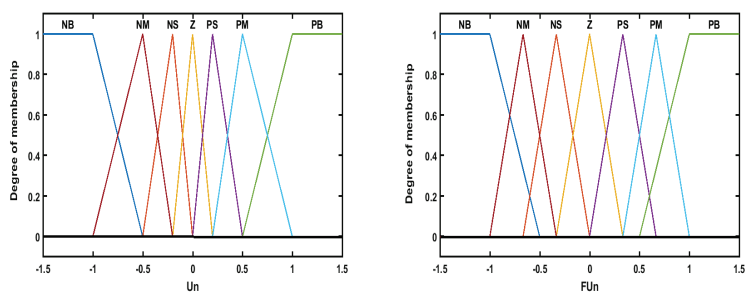

Fig. 3: Membership functions for fuzzy input ' $U_{n}$ ' and output ' $F U_{n}$ '

membership functions, namely: Negative Big (NB), Negative Medium (NM), Negative Small (NS), Zero (Z), Positive Small (PS), Positive Medium (PM), and Positive Big (PB), with the range of $[-1,1]$ for both input and output.

Considering the fuzzy input and output, the rules-base to produce the desired natural control $\left(u_{n}\right)$ can be set up as in Table I

The natural control is obtained after defuzzification process can be expressed as

$$
u_{n}=G_{u} u_{f n}
$$

where $G_{n}$ is a gain to factorise the output signal, while $u_{f n}$ represents the crisp values obtained from the FLC. Thus the overall control signal of FSMC can be written as

$$
u=-(S \hat{g}(x))^{-1} S \hat{f}(x, t)+G_{u} u_{f n}
$$

\section{NONLINEAR OBSERVER DESIGN FOR MLS}

Let, the nonlinear system be represented as

$$
\begin{aligned}
\dot{x} & =f(x)+g(x) u \\
y & =h(x)
\end{aligned}
$$

where $h(x)$ is a differentiable vector field of $x$. To design an optimal controller based on state feedback for such a nonlinear system; all states need to be measured or estimated. The object position can be measured by an appropriate sensor and coil current. The velocity measurement, however, is not

TABLE I: The Fuzzy rule base for FSMC

\begin{tabular}{|c|c|c|c|c|c|c|c|}
\hline$u_{f n}$ & $N B$ & $N M$ & $N S$ & $Z$ & $P S$ & $P M$ & $P B$ \\
\hline & $P B$ & $P M$ & $P S$ & $Z$ & $N S$ & $N M$ & $N B$ \\
\hline
\end{tabular}

straight forward. The difficulties associated with unmeasured states can be solved through a state estimation process.

A nonlinear observer can be set up as

$$
\dot{\hat{x}}=f(\hat{x})+g(\hat{x}) u+l(\hat{x})(y-h(\hat{x}))
$$

where $g(\hat{x})=\left[\begin{array}{lll}0 & 0 & 1 / L\end{array}\right]^{T}, h(\hat{x})=\hat{x}_{1}$ and $l(x)$ is nonlinear gain which can be written as

$$
l(\hat{x})=(J(\hat{x}))^{-1} G
$$

Here, $J$ is the Jacobian matrix of coordinates obtained from nonlinear coordinate transformation as follows

$$
J(\hat{x})=\left[\begin{array}{ccc}
1 & 0 & 0 \\
0 & 1 & 0 \\
\frac{2 c}{m} \frac{\hat{x}_{3}^{2}}{\hat{x}_{1}^{3}} & 0 & -\frac{2 c}{m} \frac{\hat{x}_{3}}{\hat{x}_{1}^{2}}
\end{array}\right]
$$

In general, the nonlinear gain $l$ can be written as

$$
l=\left[\begin{array}{c}
G_{1} \\
G_{2} \\
\frac{\hat{x}_{3}}{\hat{x}_{1}} G 1-\frac{m}{2 c} \frac{\hat{x}_{1}^{2}}{\hat{x}_{3}} G_{3}
\end{array}\right]
$$

Therefore, the nonlinear observer for MLS system can expressed as

$$
\left.\begin{array}{rl}
{\left[\begin{array}{c}
\dot{\hat{x}}_{1} \\
\dot{\hat{x}}_{2} \\
\dot{\hat{x}}_{3}
\end{array}\right]=\left[\begin{array}{c}
\hat{x}_{2} \\
g-\frac{c}{m}\left(\frac{\hat{x}_{3}}{\hat{x}_{1}}\right)^{2} \\
-\frac{R}{L} \hat{x}_{3}+\frac{2 c}{L} \frac{\hat{x}_{2} \hat{x}_{3}}{\hat{x}_{1}^{2}}
\end{array}\right]+\left[\begin{array}{c}
0 \\
0 \\
\frac{1}{L}
\end{array}\right] u} \\
+ \\
G_{1} \\
G_{2} \\
\frac{\hat{x}_{3}}{\hat{x}_{1}} G 1-\frac{m}{2 c} \frac{\hat{x}_{1}^{2}}{\hat{x}_{3}} G_{3}
\end{array}\right]\left(\begin{array}{c}
0 \\
\left.x_{1}-\hat{x}_{1}\right)
\end{array}\right.
$$

Verification of this kind of observer is considered as a difficult task as it requires that the right side of equation (3) should ascertain the global Lipschitz condition. For this reason this type of observer is considered only as local stable observer, which means that the estimation error dynamics of equation (17) should have a finite escape time (observer error converges to zero within a finite time). Thus in this paper the gains $\mathrm{G}$ were constructed in such a way, that the observer dynamics (High-Gain Observer HGO) are much faster than the system dynamics (at least five-times). 
The estimated states which are obtained from the nonlinear observer are used to implement the control law of the exact linearizing controller (see Fig. 4).

\section{Simulation RESUlts}

A block diagram representation of active magnetic levitation with the proposed controller is shown in Fig. 3. Simulations were carried out with the following values for the system parameters: $\mathrm{M}=21.2 \mathrm{e}-3 \mathrm{~kg}, \mathrm{C}=8.248 * 1 \mathrm{e}-5$, $\mathrm{N}^{*} \mathrm{~m} / \mathrm{A}^{\wedge} 2, \mathrm{R}=4.2 \Omega, \mathrm{L}=0.02 \mathrm{H}$. The linear gain $\mathrm{G}$ was calculated according to the formula with the value of $\mathrm{a}=50$, that was used in [8] the form of

$$
G=\left[\begin{array}{c}
6 a \\
12 a^{2} \\
8 a^{3}
\end{array}\right]
$$

Initially, the set point control for the system was located at $15 \mathrm{~mm}$ with initial position of $x_{1 e}=18 \times 10^{-3} \mathrm{~m}$, $x_{2 e}=0 \mathrm{~m} / \mathrm{s}$ and the obtained initial current as $x_{3 e}=$ $\sqrt{m \times g \times 18 \times 10^{-3}}=0.9039$ A. Fig. 5 shows the performances of 'SMC and FSMC. It is noted that both controllers could stabilise the object and track the set point control and the response rise time was shorter with FSMC. Moreover, FSMC generated much smoother control signal than SMC as shown in Fig. 6

Table II explores the effect of uncertainty in the mass of object in the system with set-point of $15 \mathrm{~mm}$, as the mass was decreased to the limit of $70 \%$ and increased up to around 255\%. It is noted that FSMC achieved the minimum Integral Absolute Error (IAE) as well as minimum Mean Square Error (MSE).

Step responses of the magnetic ball system with these two controllers are shown in Figures 7-10 in presence of different percentage mass uncertainties.

Figures 11 and 12 show the control efforts of SMC and FSMC with mass percentages of $80 \%$ and $120 \%$ respectively. As it can FSMC delivered a smooth the control signal and overcome the phenomena of chartering.

\section{CONCLUSION}

A nonlinear full-order observer-based controller with fuzzy sliding-mode controller has been proposed FSMC to stabilise an active magnetic levitation system. The performance of

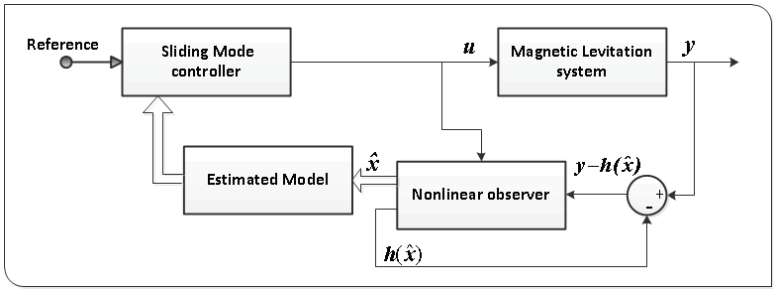

Fig. 4: Overall Maglev system

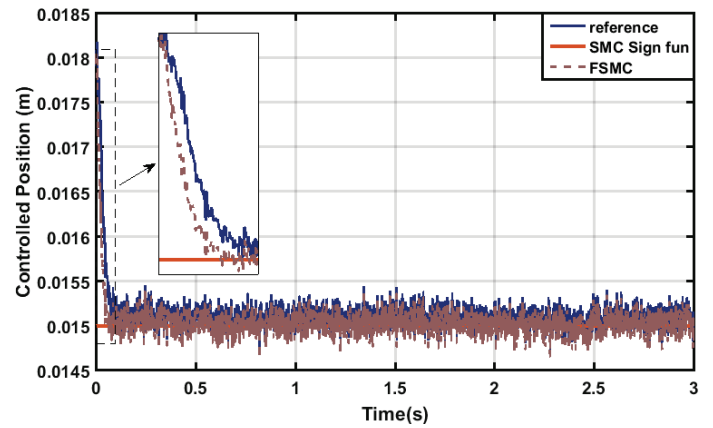

Fig. 5: System response using SMC and FSMC with set-point of $15 \mathrm{~mm}$

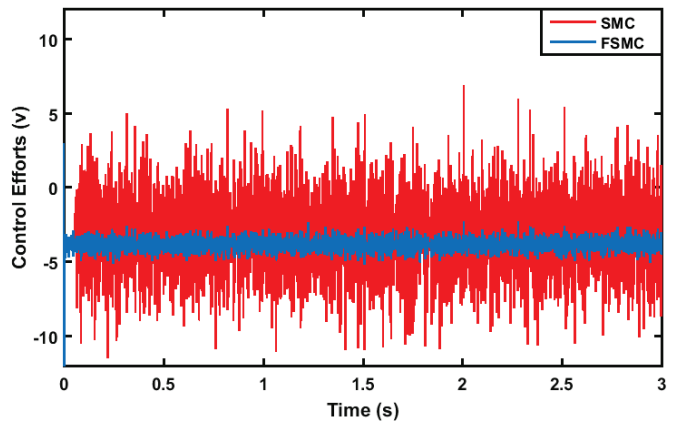

Fig. 6: The control effort of SMC and FSMC controllers with set-point of $15 \mathrm{~mm}$

TABLE II: The performance indices for the control schemes with set-point of $15 \mathrm{~mm}$

\begin{tabular}{|c|c|c|c|c|}
\hline \multirow{2}{*}{$\begin{array}{l}\text { mass uncertainty } \\
\qquad(\mathrm{m} \%)\end{array}$} & \multicolumn{2}{|r|}{ SMC } & \multicolumn{2}{|c|}{ FSMC } \\
\hline & IAE & MSE & IAE & MSE \\
\hline 70 & - & - & 16.405 & $8.03 \times 10^{-3}$ \\
\hline $78^{*}$ & 27.398 & $4.46 \times 10^{-3}$ & - & - \\
\hline 80 & 27.478 & $4.24 \times 10^{-4}$ & 10.959 & $5.56 \times 10^{-4}$ \\
\hline 90 & 13.933 & $3.23 \times 10^{-4}$ & 5.631 & $3.32 \times 10^{-4}$ \\
\hline 105 & 5.284 & $3.94 \times 10^{-4}$ & 2.414 & $2.85 \times 10^{-4}$ \\
\hline 110 & 9.157 & $5.11 \times 10^{-4}$ & 6.168 & $3.81 \times 10^{-4}$ \\
\hline 120 & 11.095 & $9.33 \times 10^{-4}$ & 10.966 & $6.21 \times 10^{-4}$ \\
\hline $122.3^{*}$ & 28.344 & $2.70 \times 10^{-3}$ & - & - \\
\hline 130 & - & - & 15.848 & $2.81 e-1$ \\
\hline 150 & - & - & 25.848 & 3.61 \\
\hline 180 & - & - & 41.334 & 8.03 \\
\hline 200 & - & - & 51.805 & 9.98 \\
\hline 250 & - & - & 78.624 & 12.95 \\
\hline $255.5 *$ & - & - & 81.377 & 13.17. \\
\hline
\end{tabular}

proposed FSMC has been assessed in comparison to that of conventional, and it has been demonstrated that FSMC has stabilised the system in the presence of high mass uncertainty of up to $155 \%$ 'that is mass percentage of $255 \%$ ', as compared to SMC which could stabilise the system only up to the limit of mass uncertainty of around $23 \%$. The results show that the generated control signals using FSMC are much smoother than those with SMC, and this has improved the regulation and quality of control provided by FSMC with 


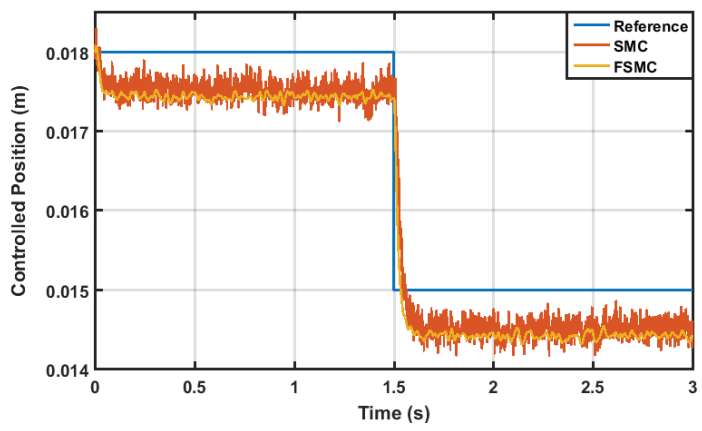

Fig. 7: System response in the presence of mass uncertainty, $\mathrm{m}=80 \%$

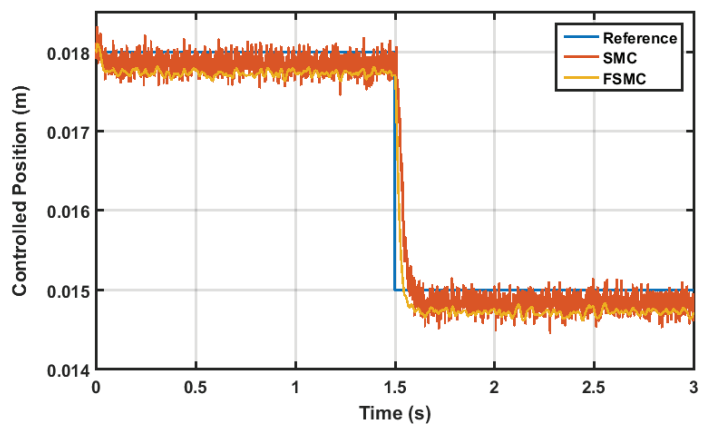

Fig. 8: System response in the presence of mass uncertainty, $\mathrm{m}=90 \%$

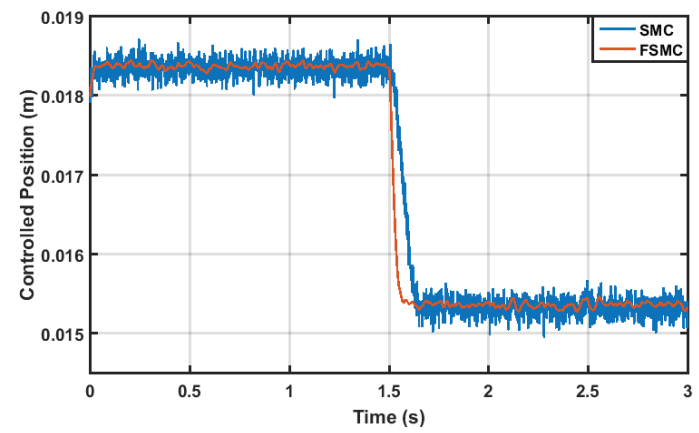

Fig. 9: System response in the presence of mass uncertainty, $\mathrm{m}=110 \%$

nonlinear observer. Future work will address experimental implementation using the proposed control schemes.

\section{REFERENCES}

[1] C. Aravind, S. Tay, M. Norhisam, I. Aris, and J. Kumar, "Reduced mechanical oscillations using the maglev concept in vertical axis wind turbine," in Renewable Energy and Sustainable Energy (ICRESE), 2013 International Conference on. IEEE, 2013, pp. 248-252.

[2] F. Song and S. M. Smith, "A comparison of sliding mode fuzzy controller and fuzzy sliding mode controller," in Fuzzy Information Processing Society, 2000. NAFIPS. 19th International Conference of the North American. IEEE, 2000, pp. 480-484.

[3] C.-L. Kuo, T.-H. S. Li, and N. R. Guo, "Design of a novel fuzzy sliding-mode control for magnetic ball levitation system," Journal of Intelligent and Robotic Systems, vol. 42, no. 3, pp. 295-316, 2005.

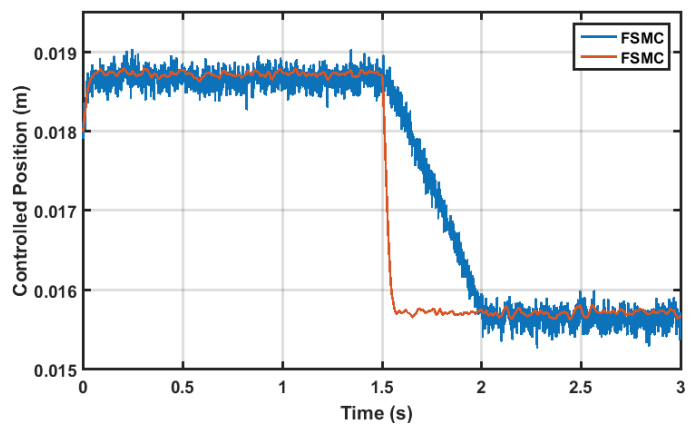

Fig. 10: System response in the presence of mass uncertainty, $\mathrm{m}=120 \%$

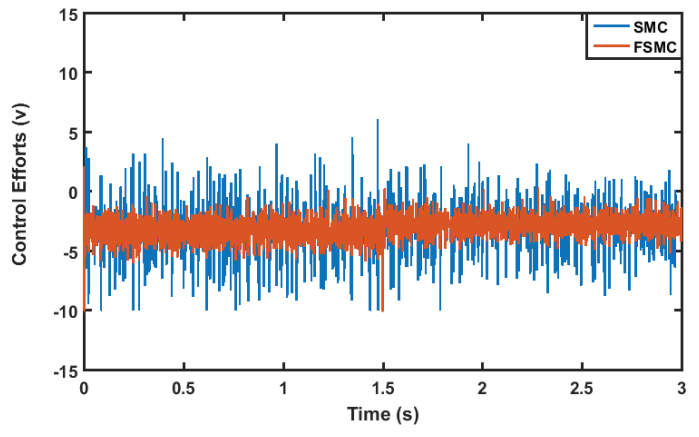

Fig. 11: The control effort of SMC \& FSMC with mass percentage of $80 \%$

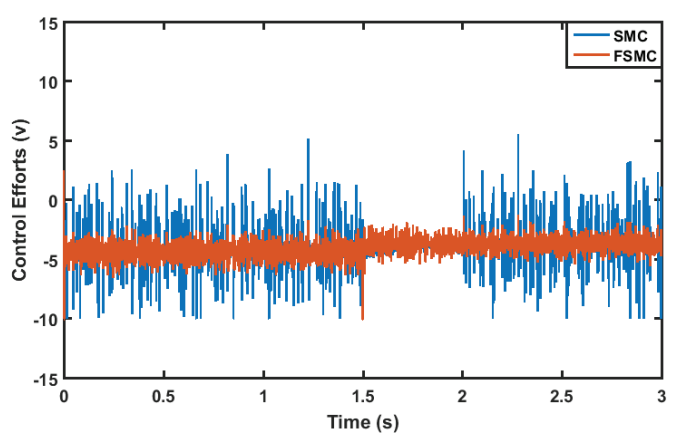

Fig. 12: The control effort of SMC \& FSMC with mass percentage of $120 \%$

[4] N. Al-Muthairi and M. Zribi, "Sliding mode control of a magnetic levitation system," Mathematical Problems in Engineering, vol. 2004, no. 2, pp. 93-107, 2004.

[5] J.-J. E. Slotine, W. Li et al., Applied nonlinear control. Prentice-hall Englewood Cliffs, NJ, 1991, vol. 199, no. 1.

[6] R. DeCarlo, S. H. Zak, G. P. Matthews et al., "Variable structure control of nonlinear multivariable systems: a tutorial," Proceedings of the IEEE, vol. 76, no. 3, pp. 212-232, 1988.

[7] A. R. FIRDAUS and M. TOKHI, "Sliding mode control-based interval type-2 fuzzy logic controller for quadcopter uavs," in ASSISTIVE ROBOTICS: Proceedings of the 18th International Conference on CLAWAR 2015. World Scientific, 2016, pp. 555-563.

[8] A. M. Benomair and M. O. Tokhi, "Nonlinear full-order observerbased controller design for active magnetic levitation via lqr-feedback linearization," in press. 
[9] A. Benomair, F. Bashir, and M. Tokhi, "Optimal control based lqrfeedback linearisation for magnetic levitation using improved spiral dynamic algorithm," in Methods and Models in Automation and Robotics (MMAR), 2015 20th International Conference on. IEEE, 2015, pp. 558-562.

[10] A. M. Benomair and M. O. Tokhi, "Control of single axis magnetic levitation system using fuzzy logic control," in Science and Information Conference (SAI), 2015, July 2015, pp. 514-518. 